\title{
Влияние лантана на транспортные свойства оксиселенидов $\mathrm{Bi}_{1-x} \mathrm{La}_{x} \mathrm{CuSeO}$
}

\author{
(С) Д.С. Панкратова ${ }^{1}$, А.П. Новицкий ${ }^{1}$, К.В. Кусков $^{1}$, И.А. Сергиенко ${ }^{1}$, Д.В. Лейбо ${ }^{1}$, \\ А.Т. Бурков ${ }^{2}$, П.П. Константинов ${ }^{2}$, В.В. Ховайло ${ }^{1}$ \\ ${ }^{1}$ Национальный исследовательский технологический университет „МИСиС“, \\ 119049 Москва, Россия \\ ${ }^{2}$ Физико-технический институт им. А.Ф. Иофффе Российской академии наук, \\ 194021 Санкт-Петербург, Россия \\ E-mail: d.pankratova@misis.ru
}

(Поступила в Редакцию 20 декабря 2018 г.

В окончательной редакции 24 декабря 2018 г.

Принята к публикации 28 декабря 2018 г.)

Представлены результаты исследования транспортных свойств оксиселенидов химического состава $\mathrm{Bi}_{1-x} \mathrm{La}_{x} \mathrm{CuSeO}(x=0.02,0.04,0.06)$-типа проводимости. По температурным зависимостям удельного электросопротивления, концентрации и подвижности носителей заряда было выявлено, что замещение ионов $\mathrm{Bi}^{3+}$ на ионы $\mathrm{La}^{3+}$ приводит к увеличению концентрации носителей заряда, предположительно, за счет генерации дырок, обусловленной формированием вакансий висмута с увеличением степени замещения.

DOI: 10.21883/FTP.2019.05.47552.10

\section{1. Введение}

В последние несколько лет оксидные термоэлектрические материалы привлекают значительное внимание научного сообщества за счет их низкой стоимости, нетоксичных элементов, входящих в состав, высокой термической и химической стабильности [1-4]. Оксихалькогениды химического состава $\mathrm{LnCuChO}$ (где Ln трехвалентные ионы $\mathrm{La}, \mathrm{Bi}, \mathrm{Nd}$ и др.; $\mathrm{Ch}$ - ионы халькогенидов, такие как $\mathrm{S}, \mathrm{Se}$ или Те) кристаллизуются по структурному типу $\mathrm{ZrCuSiAs}$ с пространственной группой $P 4 / \mathrm{nmm}$. Их кристаллическая структура состоит из слоев $\left(\mathrm{Cu}_{2} \mathrm{Ch}_{2}\right)^{2-}$, чередующихся со слоями $\left(\mathrm{Ln}_{2} \mathrm{O}_{2}\right)^{2+}$ вдоль оси $c$ тетрагональной ячейки. Слои $\left(\mathrm{Cu}_{2} \mathrm{Ch}_{2}\right)^{2-}$ являются проводящими слоями, управляя свойствами которых можно достигать высоких значений электропроводности, в то время как слои $\left(\mathrm{Ln}_{2} \mathrm{O}_{2}\right)^{2+}$ способствуют сильному рассеянию фононов, что приводит к низким значениям теплопроводности для соединений этого семейства [5-10]. Одними из наиболее перспективных в последнее время считаются оксиселениды химического состава $\mathrm{BiCuSeO}$, которые обладают высокими термоэлектрическими характеристиками благодаря сочетанию природно низкой теплопроводности и относительно высоких значений коэффициента термоэдс и электропроводности [11-13]. Это обусловлено более узкой запрещенной зоной по сравнению с соединениями, в которых на месте Ві находится ион редкоземельного элемента [14-16].

В данной работе было исследовано влияние замещения ионов $\mathrm{Bi}^{3+}$ ионами $\mathrm{La}^{3+}$ на транспортные свойства оксиселенида $\mathrm{Bi}_{1-x} \mathrm{La}_{x} \mathrm{CuSeO}(x=0.02,0.04$, $0.06)$ в интервале температур 80-473 К. В предыдущих работах было показано, что дно зоны проводимости преимущественно определяется $6 p$-орбиталями атомов висмута, в то время как потолок валентной зоны обусловлен гибридизацией между $3 d$-орбиталями меди и $4 d$-орбиталями селена $[15,17,18]$. Изменять положение дна зоны проводимости можно с помощью замещения ионов $\mathrm{Bi}^{3+}$ редкоземельными ионами $\mathrm{Ln}^{3+}$, более того, было показано что подобное замещение приводит к увеличению ширины запрещенной зоны и увеличению подвижности носителей заряда [19].

\section{2. Образцы и методы исследования}

Образцы химического состава $\mathrm{Bi}_{1-x} \mathrm{La}_{x} \mathrm{CuSeO}(x=0$, $0.02,0.04$ и 0.06$)$ были синтезированы методом двухступенчатого твердофазного синтеза с последующим спеканием методом искрового плазменного спекания. В качестве исходных реагентов были использованы мелкодисперсные порошки $\mathrm{Bi}_{2} \mathrm{O}_{3}, \mathrm{Bi}, \mathrm{Cu}, \mathrm{Se}$ и $\mathrm{La}_{2} \mathrm{O}_{3}$. Стехиометрическая смесь порошков была смешана при помощи планетарной микромельницы (Fritsch Pulverisette 7 premium line), после чего образцы были спрессованы при комнатной температуре и подвергались отжигу в запаянной кварцевой ампуле в течение 8 ч при температуре $573 \mathrm{~K}$ в вакууме. После первой ступени синтеза был проведен размол полученных образцов, которые затем вновь подвергались холодному прессованию и отжигу при температуре $973 \mathrm{~K}$ в течение 12 ч в запаянной кварцевой ампуле. С целью получения объемных образцов высокой плотности, полученные образцы измельчались в планетарной микромельнице в течение 8 ч в атмосфере аргона со скоростью 300 об/мин. Затем проводилось искровое плазменное спекание (SPS Labox 650, Sinter Land) порошков в графитовой пресс-форме диаметром 12.7 мм в течение 5мин при одноосном давлении 50 МПа и температуре $973 \mathrm{~K}$, скорость нагрева 
и охлаждения были 50 и $20 \mathrm{~K} /$ мин соответственно. Полученные объемные образцы в форме цилиндра отжигались в атмосфере аргона в течение 6 ч при $973 \mathrm{~K}$.

Фазовый состав был исследован методом рентгеновской дифракции на дифрактометре Дифрей 401 с использованием излучения $\mathrm{Cr}_{\alpha}(\lambda=2.2911 \AA)$. Погрешность при определении фазового состава не превышала $5 \%$. Концентрация и подвижность носителей заряда определялись из измерений эффекта Холла по формулам $p=1 / e R_{\mathrm{H}}$ и $\mu=\sigma R_{\mathrm{H}}$, где $p-$ концентрация носителей заряда $\left(\mathrm{cm}^{-3}\right), R_{\mathrm{H}}-$ константа Холла $\left(\mathrm{cm}^{3} \cdot \mathrm{K}^{-1}\right), \mu-$ подвижность носителей заряда $\left(\mathrm{cm}^{2} \cdot \mathrm{B}^{-1} \cdot \mathrm{c}^{-1}\right), \sigma-$ электропроводность $\left(\mathrm{OM}^{-1} \cdot \mathrm{cm}^{-1}\right)[20,21]$.

\section{3. Результаты исследований}

На рис. 1 представлены дифрактограммы образцов $\mathrm{Bi}_{1-x} \mathrm{La}_{x} \mathrm{CuSeO}(x=0.02,0.04,0.06)$ после отжига. Все основные пики могут быть проиндексированы как характерные пики $\mathrm{BiCuSeO}$ со структурой $\mathrm{ZrSiCuAs}$.

На рис. 2 представлены зависимости удельного электросопротивления от температуры. Для всех образцов наблюдался рост электросопротивления с ростом температуры. Из рис. 2 видно, что с ростом концентрации лантана значение электросопротивления снижается во всем интервале температур, что может быть связано с формированием дополнительных вакансий висмута при увеличении степени замещения [19,22-24]. Кроме этого, при высоких температурах значения холловской подвижности носителей заряда для всех образцов являются практически одинаковыми (рис. 3), в то время как концентрация носителей заряда возрастает с увеличением содержания лантана (рис. 4).

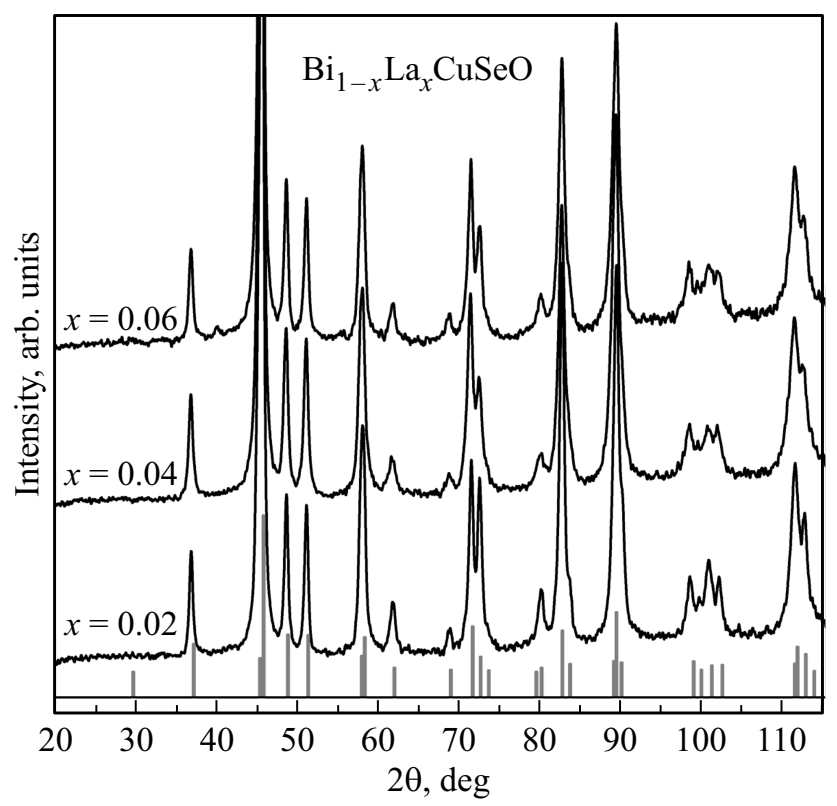

Рис. 1. Дифрактограммы образцов $\mathrm{Bi}_{1-x} \mathrm{La}_{x} \mathrm{CuSeO}(x=0.02$, $0.04,0.06)$.

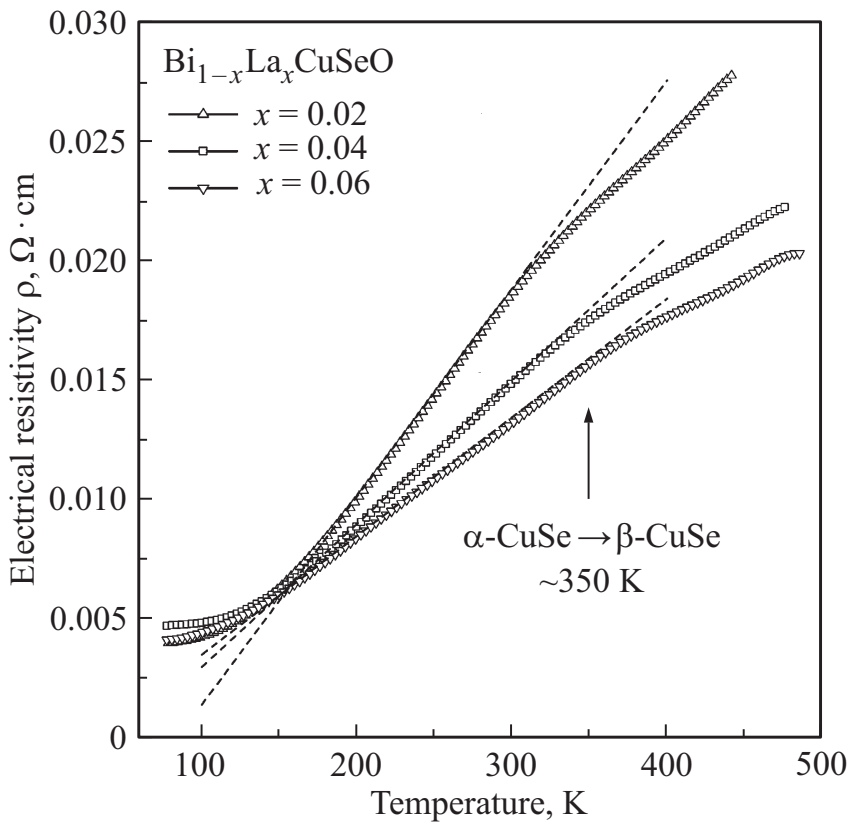

Рис. 2. Зависимость удельного электросопротивления от температуры для $\mathrm{Bi}_{1-x} \mathrm{La}_{x} \mathrm{CuSeO}(x=0.02,0.04,0.06)$.

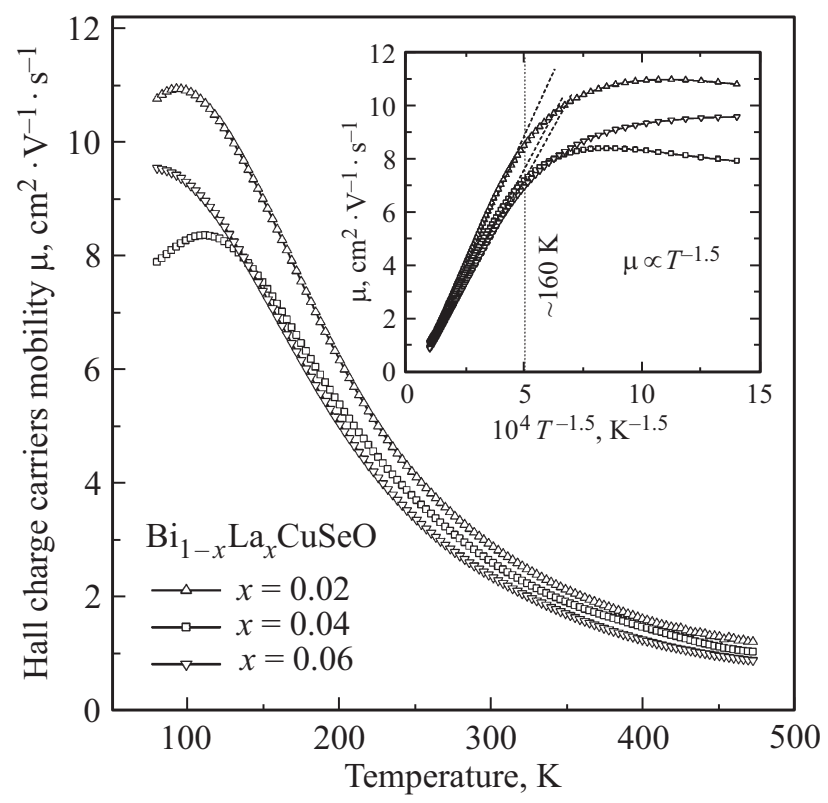

Рис. 3. Температурная зависимость подвижности носителей заряда для $\mathrm{Bi}_{1-x} \mathrm{La}_{x} \mathrm{CuSeO}(x=0.02,0.04,0.06)$. На вставке - температурная зависимость подвижности в координатах $\mu-T^{1.5}$.

По нашему мнению, такое изменение транспортных свойств $\mathrm{Bi}_{1-x} \mathrm{La}_{x} \mathrm{CuSeO}$ может быть обусловлено возникновением дополнительных вакансий висмута при его замещении лантаном, как это было показано в других работах [22-25]. Наряду со снижением энергии формирования вакансий Ві при допировании исходного соединения (см., например, [25]) наличие фазы CuSe также может 


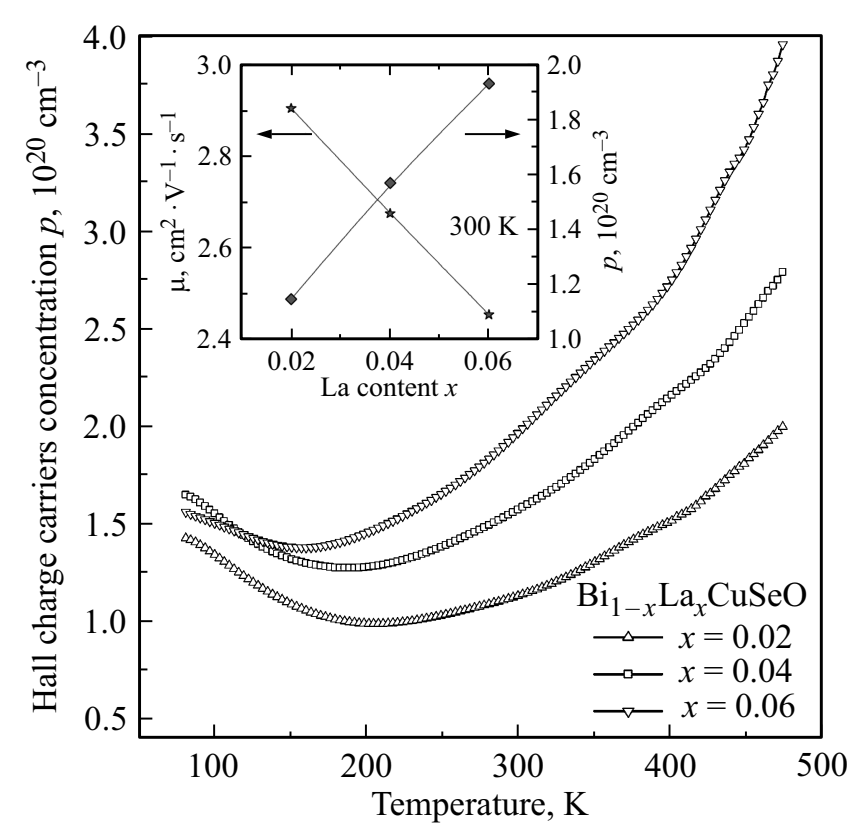

Рис. 4. Температурная зависимость концентрации носителей заряда для $\mathrm{Bi}_{1-x} \mathrm{La}_{x} \mathrm{CuSeO}(x=0.02,0.04,0.06)$. На вставке концентрационные зависимости подвижности и концентрации носителей заряда при $300 \mathrm{~K}$.

способствовать возникновению дополнительных вакансий висмута, как было показано в работе [24]. Несмотря на то, что рентгенофазовый анализ (рис. 1) указывает на то, что все образцы являются однофазными, косвенным подтверждением наличия примесной фазы $\mathrm{CuSe}$ в $\mathrm{Bi}_{1-x} \mathrm{La}_{x} \mathrm{CuSeO}$ является изменение наклона кривых электросопротивления для всех образцов в окрестности $350 \mathrm{~K}$, что, по-видимому, соответствует структурному фазовому переходу из $\alpha$ в $\beta$ фазу CuSe (рис. 2). Однако стоит отметить, что количество данной фазы не превышает 5\% (рис. 1). Учитывая то, что $\mathrm{BiCuSeO}$ является полупроводниковым соединением $p$-типа, увеличение количества вакансий висмута приводит к увеличению концентрации носителей заряда, так как каждая вакансия висмута генерирует три дырки в соответствии с реакцией $2 \mathrm{Bi}_{1-x} \mathrm{CuSeO} \rightarrow\left(\mathrm{Bi}_{2-2 x} \mathrm{O}_{2}\right)^{2(1-3 x)+}$ $+\left(\mathrm{Cu}_{2} \mathrm{Se}_{2}\right)^{2-}+6 x h^{+}$. Стоит также отметить, что для всех образцов при температурах выше $160 \mathrm{~K}$ преобладает рассеяние носителей заряда на акустических фононах (см. вставку на рис. 3).

Соединения данного семейства чувствительны к стехиометрии и их транспортные свойства могут сильно варьироваться за счет образования дефектов или вакансий по меди, висмуту или кислороду [22-24,26-28]. Более того, наличие вакансий может сильно зависеть от метода синтеза и выбора параметров термической обработки. Полученные в данной работе результаты показывают, что уменьшение электросопротивления обусловлено ростом концентрации носителей заряда (см. вставку на рис. 4), предположительно, вызванного образованием вакансий висмута.

\section{4. Заключение}

Исследованы транспортные свойства объемных образцов оксиселенидов $\mathrm{Bi}_{1-x} \mathrm{La}_{x} \mathrm{CuSeO}(x=0.02,0.04,0.06)$ $p$-типа проводимости в интервале температур 80-473 K. Было выявлено, что замещение ионов $\mathrm{Bi}^{3+}$ на ионы $\mathrm{La}^{3+}$ приводит к уменьшению удельного электросопротивления в результате роста концентрации носителей заряда, в свою очередь обусловленного формированием вакансий висмута при увеличении степени замещения.

Работа выполнена при финансовой поддержке Министерства образования и науки Российской Федерации в рамках программы повышения конкурентоспособности НИТУ „МИСиС“.

\section{Список литературы}

[1] C. Barreteau, D. Berardan, N. Dragoe. J. Solid State Chem., 222, 53 (2015).

[2] F. Li, T.-R. Wei, F. Kang, J.-F. Li. J. Mater. Chem. A, 1, 11942 (2013).

[3] J.W. Fergus. J. Eur. Ceram. Soc., 32, 525 (2012).

[4] Y. Yin, B. Tudu, A. Tiwari. Vacuum., 146, 356 (2017).

[5] K. Ueda, K. Takafuji, H. Hosono. J. Solid State Chem., 170, 182 (2003).

[6] H. Ohta, S. Kim, Y. Mune, T. Mizoguchi, K. Nomura, S. Ohta, T. Nomura, Y. Nakanishi, Y. Ikuhara, M. Hirano, H. Hosono, K. Koumoto. Nature Materials, 6, 129 (2007).

[7] K. Ueda, H. Hosono, N. Hamada. J. Appl. Phys., 98, 043506 (2005).

[8] K. Ueda, K. Takafuji, H. Yanagi, T. Kamiya, H. Hosono, H. Hiramatsu, M. Hirano, N. Hamada. J. Appl. Phys., 102, 113714 (2007).

[9] K. Ueda, K. Takafuji, H. Hiramatsu, H. Ohta, T. Kamiya, M. Hirano, H. Hosono. Chem. Mater., 15, 3692 (2003).

[10] R. Pöttgen, D. Johrendt. Zeitschrift fur Naturforsch. Sect. B, J. Chem. Sci., 63, 1135 (2008).

[11] F. Li, J.-F. Li, L.-D. Zhao, K. Xiang, Y. Liu, B.-P. Zhang, Y.-H. Lin, C.-W. Nan, H.-M. Zhu. Energy Environ. Sci., 5, 7188 (2012).

[12] L.D. Zhao, D. Berardan, Y.L. Pei, C. Byl, L. Pinsard-Gaudart, N. Dragoe. Appl. Phys. Lett., 97, 092118 (2010).

[13] L.-D. Zhao, J. He, D. Berardan, Y. Lin, J.-F. Li, C. Nan, N. Dragoe. Energy Environ. Sci., 7, 2900 (2014).

[14] C. Barreteau, L. Pan, E. Amzallag, L.D. Zhao, D. Bérardan, N. Dragoe. Semicond. Sci. Technol., 29, 064001 (2014).

[15] H. Hiramatsu, H. Yanagi, T. Kamiya, K. Ueda. Chem. Mater., 20, 326 (2008).

[16] M. Yasukawa. J. Appl. Phys., 95, 3594 (2004).

[17] K. Ueda, H. Hosono, N. Hamada. J. Phys. Condens. Matter, 16, 5179 (2004).

[18] K. Ueda, H. Hiramatsu, H. Ohta, M. Hirano, T. Kamiya, H. Hosono. Phys. Rev. B, 69, 155305 (2004).

[19] Y. Liu, J. Ding, B. Xu, J. Lan, Y. Zheng, B. Zhan, B. Zhang, Y. Lin, C. Nan. Appl. Phys. Lett., 106, 233903 (2015).

[20] Б.Ф. Грузинов, П.П. Константинов. ПТЭ, 5, 225 (1972).

[21] M.V. Vedernikov, P.P. Konstantinov, A.T. Burkov. Eighth Int. Conf. Thermoelectr. Energy Conversion, July 10-13 (France, Nancy, 1989) p. 45. 
[22] Z. Li, C. Xiao, S. Fan, Y. Deng, W. Zhang, B. Ye, Y. Xie. J. Am. Chem. Soc., 137, 6587 (2015).

[23] S. Das, A. Ramakrishnan, K. Chenю J. Phys. D: Appl. Phys., 51, 035501 (2018).

[24] M. Ishizawa, Y. Yasuzato, H. Fujishiro, T. Naito, H. Katsui, T. Goto. J. Appl. Phys., 123, 245104 (2018).

[25] T.-H. An, Y.S. Lim, H.-S. Choi, W.-S. Seo, C.-H. Park, G.-R. Kim, C. Park, C.H. Lee, J.H. Shim. J. Mater. Chem. A, 2, 19759 (2014).

[26] Y. Liu, L.D. Zhao, Y. Liu, J. Lan, W. Xu, F. Li, B.P. Zhang, D. Berardan, N. Dragoe, Y.H. Lin, C.W. Nan, J.F. Li, H. Zhu. J. Am. Chem. Soc., 133, 20112 (2011).

[27] B. Feng, G. Li, Z. Pan, X. Hu, P. Liu, Y. Li, Z. He, X. Fan. J. Ceram. Soc. Jpn, 126, 699 (2018).

[28] B. Zhan, Y. Liu, X. Tan, J. Lan, Y. Lin, C.-W. Nan. J. Am. Ceram. Soc., 98, 2465 (2015).

Редактор А.Н. Смирнов

\section{Effect of La doping on the transport properties of $\mathrm{Bi}_{1-x} \mathrm{La}_{x} \mathrm{CuSeO}$ oxyselenides}

D.S. Pankratova ${ }^{1}$, A.P. Novitskii ${ }^{1}$, K.V. Kuskov' ${ }^{1}$, I.A. Serhiienko ${ }^{1}$, D.V. Leybo ${ }^{1}$, A.T. Burkov' ${ }^{2}$, P.P. Konstantinov ${ }^{2}$, V.V. Khovaylo ${ }^{1}$

${ }^{1}$ National University of Science and Technology „MISIS“,

119049 Moscow, Russia

2 loffe Institute,

194021 St. Petersburg, Russia

Abstract In this work we present the results of the transport properties study for $p$-type $\mathrm{Bi}_{1-x} \mathrm{La}_{x} \mathrm{CuSeO}(x=0.02,0.04,0.06)$ oxyselenides. Accordingly to temperature dependencies of the electrical resistivity, charge carriers concentration and mobility, it was revealed that the substitution of $\mathrm{Bi}^{3+}$ ions to $\mathrm{La}^{3+}$ ions leads to charge carriers concentration enhancement caused by generation of holes, which is presumably attributed to the bismuth deficiencies formation with respect to the substitution level. 